\title{
Design Recommendations for a Reliable Body-Worn Patient Monitoring and Alarming Service
}

\author{
Yohanes Baptista Dafferianto Trinugroho, Rune Fensli, Frank Reichert \\ Faculty of Engineering and Science \\ University of Agder \\ Grimstad, Norway \\ \{dafferianto.trinugroho, rune.fensli, frank.reichert\}@uia.no
}

\begin{abstract}
Telehealth has been widely used in recent years to provide healthcare services at a distance. It supports eliminating space barriers and improves access to healthcare services. One important feature of telehealth is that it can be used to save lives in critical and emergency situations by means of alarming service. As health monitoring and alarming services rely quite heavily on body-worn devices/sensors for health-related patient data gathering, communications reliability between those devices and back-end monitoring and alarming server is of high importance to be taken into consideration during the design phase of such services. A slight miscalculation may cost precious human lives. This paper aims to provide design recommendations for a reliable patient monitoring and alarming service that gathers patient data mainly through body-worn sensors.
\end{abstract}

Categories and Subject Descriptors

J.3 [Computer Applications]: Life and Medical Sciences; C.2.4 [Computer Systems Organization]: Distributed Systems

\section{General Terms}

Management, Design, Reliability

\section{Keywords}

Telehealth, Patient Monitoring, Alarming Service, Reliability

\section{INTRODUCTION}

Decreasing number of birthrate and improved quality of healthcare have resulted in growing number of elderly population in many developed countries. Statistics Norway stated that there were around 625,000 people older than 67 years old living in Norway in 2010, and this number is expected to double in 2060 [1]. This trend, however, is not balanced with appropriate increase in number of healthcare workers, which causes an increasing gap between demand and supply within healthcare arena. Telehealth comes into scene as one solution to tackle the aforementioned issues by utilising information and communications technology to provide medical information and healthcare services when the provider of care and patient are separated by distance [2]. It reduces the overall cost of healthcare services by moving nonurgent treatment to patient's home, which cuts hospitalisation and transportation costs among others, and also reduces the mobility frequency of healthcare workers to/from patient's home. A study showed that telehealth can increase patient satisfaction as well, mainly due to patient's feeling of having more control of the treatments [3].

An important application of telehealth is remote patient monitoring as it provides measurements and reports of daily activities of patients to distantly located healthcare providers. Patient monitoring systems incorporate alarming subsystem that is useful in emergency situations to alert responsible personnel (e.g. doctors, nurses) in charge of the corresponding monitored patient. The emergency alarms are triggered based on the situation and condition of the patient, where the data are gathered from various different devices/sensors in the patient's surroundings. Most of these devices are worn by the patient, especially the ones which measure vital information. Some examples of these devices are electrocardiogram (ECG), pulse oximeter, and blood pressure meter. The advances of wireless communications technologies have enabled wire reduction in such devices around the patient's body, forming so-called wireless body area networks (WBAN). A typical WBAN architecture consists of a set of wearable on-body physiological sensors that forward measurements data to a personal server [4] that can be deployed either in a home gateway when the patient stays at home, or in a handheld device (e.g. smartphone) [5]. This personal server or application agent is responsible for relaying the gathered information to the remote monitoring and alarming server. Since many of patient monitoring and alarming services are built based on Internet Protocol (IP) technology, these services rely heavily on Internet connectivity between the application agent and the back-end monitoring and alarming server. This implies a stringent requirement for alwayson Internet connectivity, especially to support the alarming service for emergency situations.

This paper presents some recommendations for development and deployment of a reliable end-to-end IP-based patient monitoring and alarming service where the information is mainly gathered through body-worn sensors.

\section{SYSTEM DESIGN CONSIDERATIONS}

Telehealth in general can be implemented in two different modes: real-time and store-and-forward [6]. Real-time telehealth systems require instantaneous interactions between distantly located patient and healthcare worker. High data rate is often needed to deploy this type of telehealth systems. On the other hand, storeand-forward telehealth systems store the captured data in patient's device, then forward them at a later time to back-end servers for offline assessment by responsible healthcare personnel. For remote patient monitoring and alarming service, real-time 
information delivery is important to be applied between the patient and the healthcare provider. However, a completely synchronous communication mechanism may not be the best option since some processing of gathered data may need to be carried out at the patient's side before being relayed to the backend monitoring server. Furthermore, emergency alarms occur after reasoning processes have taken place, thus, asynchronous communications model will fit better. Despite this fact, timely dissemination of monitoring and emergency information to the healthcare provider is of utmost importance. Reliability of such end-to-end service relies heavily on the communications link between the patient and the healthcare provider.

\subsection{Outdoor Environment}

From a broader perspective, data from body-worn devices' measurements can either be sent directly from the devices to the back-end server or sent through a data aggregation device which will then forward the information to the monitoring/emergency alert server. The former, as shown in Figure 1, is quite difficult to be adopted in outdoor environments where Internet connectivity is provided mainly through cellular links. This will require every device to have its own SIM card, and additional sink servers should be present to receive each device's data before being aggregated by the monitoring server.

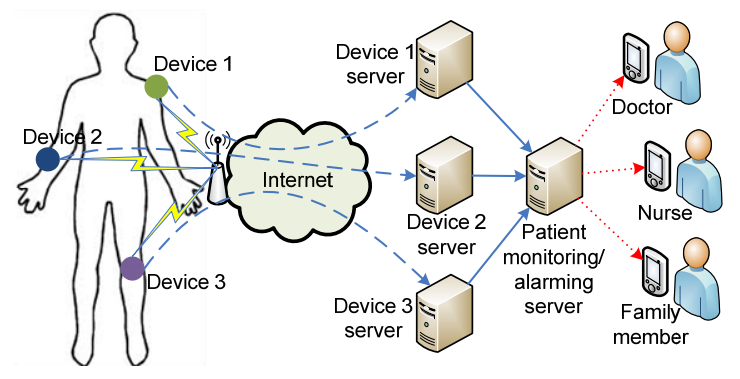

Figure 1. Direct data transmission to back-end server.

In addition, body-worn devices may not have strong specifications to perform sophisticated reasoning themselves, solely relying on data aggregation and reasoning at the back-end server. Thus, data aggregator and relay device at WBAN side approach has more advantage to be considered, as shown in Figure 2.

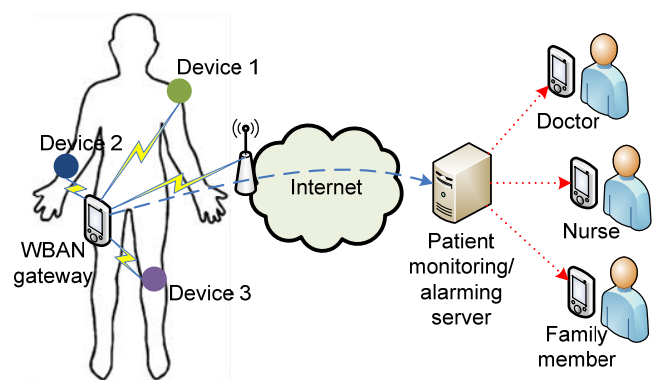

Figure 2. Brokered data transmission to back-end server.

Taking into account current smartphones' processing capabilities with more than $1 \mathrm{GHz}$ clock speed with various different communications links being supported (e.g. WLAN, GPRS, UMTS, Bluetooth, IrDA), it is possible to host personal application agent inside a smartphone to perform data aggregation and sophisticated reasoning, transforming low-level data collected from the on-body devices to high-level information, before forwarding them to the back-end server. The smartphone becomes the gateway of the WBAN in this case.

\subsection{Indoor Environment}

The architecture in Figure 1 is feasible to be adopted in indoor environments, such as in a smarthome, where the body-worn devices can send all measurements to each corresponding backend server through the smarthome's Internet gateway. However, as the patient may travel outside the smarthome environment (i.e. changing location state from indoor to outdoor), where the smarthome's Internet gateway is not reachable any longer by the body-worn devices, this architecture is not practically suitable for an indoor-outdoor roaming scenario. If this architecture is to be adopted, each body-worn device should be able to sense when it is out of reach of the smarthome's Internet gateway and use the patient's smartphone as the new gateway. This makes the architecture in Figure 2 a better option to be adopted, where the body-worn devices always use the patient's smartphone as their gateway. The location-awareness then only needs to be handled by one device (i.e. the smartphone).

In a smarthome environment, many sophisticated devices are present to assist the patient's daily life. An integration point is expected to be present as well, combining data from those devices to be used for reasoning processes. Data gathered from body-worn devices may also be used to enhance the smarthome's reasoning processes in assisting the patient at home. To achieve this, the patient's smartphone should forward the measured data from body-worn devices to the smarthome's integration platform instead of sending them to the back-end server directly. The smarthome's integration platform will then forward the information to the back-end server after conducting reasoning processes. In this case the smartphone acts only as a relay, as shown in Figure 3.

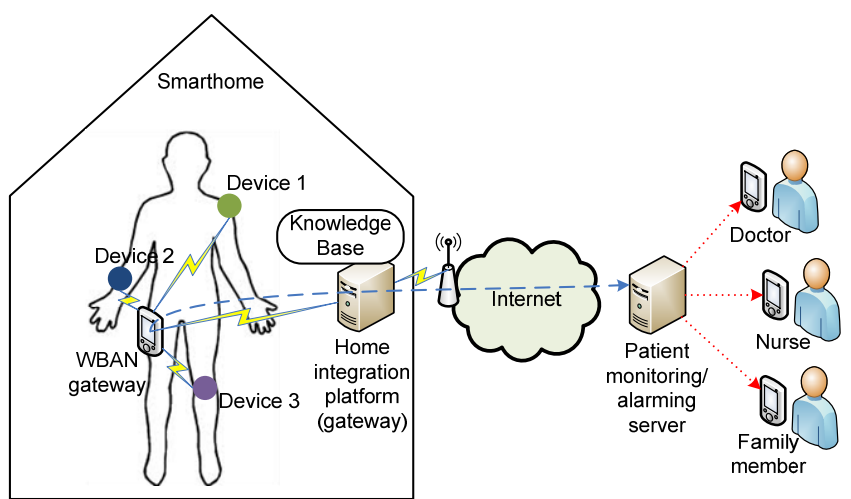

Figure 3. Indoor data transmission to back-end server.

For an indoor-outdoor roaming scenario, it is important for the smartphone to be notified or to actively sense for its location whether within the range of the smarthome's Internet gateway (i.e. inside or near the smarthome) or not. Different location positioning approaches can be employed, such as using GPS, GSM triangulation, Bluetooth, and WiFi access points. However, direct reachability test from the smartphone to the smarthome's Internet gateway is recommended, for example by sending ICMP echo request packets. In addition, a keep-alive message is recommended to be sent from the smartphone's application agent to the back-end server every certain period of time to inform the end-to-end link status, so that the back-end server can generate alarms when the communications channel is down. 


\section{COMMUNICATIONS RELIABILITY}

Reliability of communications link between a patient and healthcare provider is of great importance in telehealth services. With an assumption that communications between devices within the WBAN are reliable, this section focuses more on communications link reliability between the gateways (smartphone and smarthome) and the Internet (i.e. Internet connectivity).

\subsection{Redundancy}

Reliability of end-to-end services based on distributed system architecture as shown in previous figures depends on available communications links. Since the patient monitoring and alarming service is based on IP technology, redundant Internet connections is essential to maximise the uptime of such service. This may not be a big issue for the smarthome gateway as both fixed and wireless Internet connectivity can easily be provisioned, but it is challenging for the smartphone gateway as option for fixed connectivity does not apply, and yet redundancy in connectivity is needed to support outdoor patient monitoring and alarming service. Both smarthome and smartphone gateways are recommended to have one primary Internet connectivity link and at least one alternative/backup connection, with smarthome gateway's Internet connectivity having higher priority than smartphone's (i.e. the smartphone gateway relays all data gathered from the WBAN to the smarthome gateway whenever it is reachable). Table 1 shows possible combinations of Internet connectivity redundancy for both gateways.

Table 1. Possible connectivity redundancy combinations

\begin{tabular}{|c|c|c|c|}
\hline Priority & Gateway & Primary & Alternative/Backup \\
\hline 1 & Smarthome & xDSL & UMTS/WCDMA, \\
& & & $\begin{array}{c}\text { GPRS/EDGE, WiFi, } \\
\text { ISDN, Satellite }\end{array}$ \\
\hline 2 & Smartphone & UMTS/ & $\begin{array}{c}\text { GPRS/EDGE, } \\
\text { Satellite }\end{array}$ \\
& & WCDMA & r \\
\hline
\end{tabular}

Redundancy of connectivity between different operators of the same access networks type (i.e. roaming) is recommended as well.

\subsection{Link Quality}

The default Internet connection to support patient monitoring and alarming service should be the most stable and reliable for each gateway. However, even though Internet service providers guarantee high service level agreements (SLAs) nearing to $100 \%$ for fixed residential connections, downtime can still occur. Downtime of Internet connectivity in outdoor scenarios is expected to be more frequent. If more than one backup connectivity are available, selection between these backups needs more attention from application-layer perspective, where a smart application agent in each gateway is in charge of deciding which link to use. Link quality undoubtedly is a strong deciding factor. However, link quality consists of various parameters that need to be considered. In addition, other parameters outside link quality may need to be taken into account as well. Some widely used parameters for choosing a link are delay (round-trip time), packet loss rate, data rate, and cost. The application agent deployed in each gateway should be smart enough to take into account these parameters to make a decision of which link to use when the primary (default) link is down. In other words, the application agent should understand the context of available connectivity, including the availability of connectivity itself. This is where context-awareness is recommended to play its role as described in the next section.

\section{CONTEXT-AWARENESS IN ACTION}

Context is defined as any information that can be used to characterise the situation of an entity, where the entity can be a person, place, or any object that is relevant to the interaction between a user and an application [7]. Context-aware systems aim at automatically personalising user's environment depending on the user's context, and hence, minimising user interaction with the system and the invoked services [8]. Internet connection availability and link quality (and its parameters) are contexts as well that can be used to support patient monitoring and alarming service both inside and outside patient's home. By utilising these contexts, automatic alternative link selection process can be realised when the default connection is down.

\subsection{Ontology-based Context Modelling}

Context-aware systems depend on the context model used to formally represent the contexts as it determines the reasoning methods that can be used to perform adaptation. Ontology-based context modelling has been used quite often in recent years due to its structured and rich description of user's context that allows semantic reasoning. Ontology model is considered to have an edge over other context-modelling approaches as it enables knowledge sharing in open dynamic systems, allows an efficient reasoning on context information with well-defined declarative semantics, and enables service interoperability as well as collaborative networked services in a non-ambiguous manner [9]. Thus, ontology-based context-aware system is recommended to be incorporated by the smart agent in both gateways to maintain the knowledge base related to the patient, including the connectivity to the Internet. An ontology based on Web Ontology Language (OWL) has been developed as shown in Figure 4.

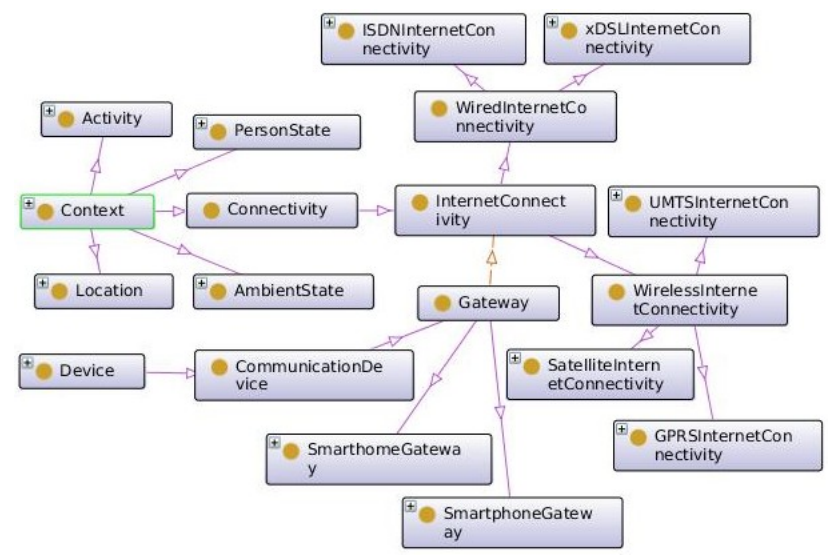

Figure 4. Ontology-based context model.

Several contexts are depicted in Figure 4, and Connectivity is one of them. This knowledge base can then be used to conduct some reasoning processes related to connectivity issues. One example of such reasoning is to make a decision when to choose a new link when the default link is down. A Semantic Web Rule Language (SWRL) is used to show this example as follows.

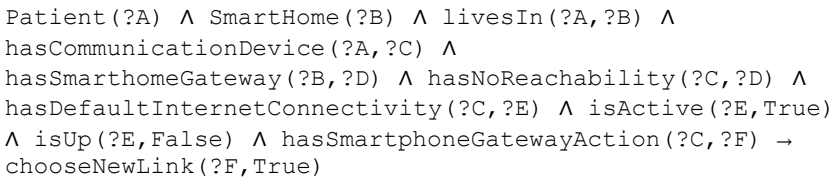


In this rule it is checked if the default Internet connectivity of the smartphone gateway is active but the connection itself is down. The reachability of the smarthome gateway from the smartphone gateway is also checked (i.e. checking whether the patient is at home or in the near vicinity), and if it is out of reach, then a new (alternative) link should be chosen.

\subsection{Link Selection}

Selecting the best available links when the default link is down may be tricky. However, it is recommended that after the reasoning processes on the ontology take place and a new link should be chosen, a link selection process should be carried out automatically by the application agent in the smartphone gateway. Different parameters such as delay (round-trip time), packet loss rate, data rate, and cost should be taken into consideration to decide which link to use. To achieve this, an API for selecting the best link should be made available for the application agent to call. This API needs to implement a link selection algorithm that can vary from a simple weighting approach to complex methods. A simple weighting algorithm is proposed to be used, explained as follows.

For all available $N$ alternative links $L=\left\{l_{1}, l_{2}, \ldots, l_{N}\right\}$, a set of $k$ parameters for $i$-th link $P_{i}=\left\{p_{i 1}, p_{i 2}, \ldots, p_{i k}\right\}$ are collected, then a weighted value $V=\left\{v_{l}, v_{2}, \ldots, v_{N}\right\}$ for each link is calculated. These values are then ranked from the biggest to the smallest, and the highest ranked link is chosen. The weights are used to distinguish the importance of each parameter, where the higher the value of the weight, the more important the parameter is. The steps are described below.

$$
\begin{array}{lc}
\hline 1: & \text { for } i \leftarrow 1 \text { to } N \text { do } \\
\text { 2: } & v_{i} \leftarrow \text { calculateWeight }\left(p_{i 1} . . p_{i k}\right) \\
\text { 3: } & v_{\text {chosen }} \leftarrow \max \left(v_{1} . . v_{N}\right) \\
\text { 4: } & \text { chosenLink } \leftarrow l_{\text {chosen }} \\
\hline
\end{array}
$$

The calculateWeight function in line 2 is defined to be sum of weighted parameter value for all parameters being used by all links (all links use same parameters). However, since some parameters such as delay (round-trip time), packet loss rate, and cost should be minimised and other parameters such as data rate should be maximised, the summation should be separated into two groups. Then subtraction between the sum of weighted value that should be maximised and the sum of weighted value that should be minimised will be calculated, as follows.

$$
v=\frac{\sum_{i=1}^{m} w_{\max _{i}} p_{\max _{i}}-\sum_{j=1}^{n} w_{\min _{j}} p_{\min _{j}}}{\sum_{i=1}^{m} w_{\max _{i}}+\sum_{j=1}^{n} w_{\min _{j}}}
$$

$m$ is the number of parameters that should be maximised and $n$ is the number of parameters that should be minimised, such that $m+n=k . w_{\max }$ and $w_{\min }$ are weights of parameters that should be maximised and minimised respectively. The denominator in equation (1) can be eliminated by normalisation, such that:

$$
\sum_{i=1}^{m} w_{\max _{i}}+\sum_{j=1}^{n} w_{\min _{j}}=1
$$

If all parameters are equally important, then the weights $w=w_{\max }=w_{\min }$ for all parameters are uniform to be $1 / k$.

In order to perform link selection, the application agent in the smartphone needs to gather the different used parameters for each available link. It is recommended that the functionality of gathering these parameters is made available by means of APIs which are called by the application agent whenever Internet connection loss through the default link is detected. This will save a lot of data traffic compared to having to query every certain period of time. One example is an API to gather delay (round-trip time) parameter that can use ICMP echo request messages to the back-end monitoring server.

\section{CONCLUSIONS}

A system design that supports indoor-outdoor roaming scenario for remote patient monitoring and alarming service is proposed and recommended in this paper. A smarthome gateway and a smartphone gateway are recommended to be used, where measurements from body-worn sensors are forwarded to the smartphone gateway first. These data will then go through some reasoning processes either in the smartphone or smarthome gateway depending on whether the smarthome gateway is reachable from the smartphone gateway. Redundancy of Internet connectivity is recommended to maintain the reliability of the provisioned service as Internet connectivity loss can occur frequently in dynamic environment. An ontology-based context modelling is proposed and recommended to be used to maintain the knowledge base of the user's contexts, which can further be used to automatically select the best available link when the main Internet connectivity is down. Finally, a simple weighting algorithm is proposed to be used for selecting the best available backup link. Evaluation of the design recommendations is planned to be conducted next.

\section{REFERENCES}

[1] Mørk, E. Seniorer i Norge 2010. Statistisk sentralbyrå. Oslo, 2011.

[2] Fitzmaurice, J. M. 2003. Telehealth Research and Evaluation: Implications for Decision Makers. In Proceedings of Pacific Medical Technology Symposium (Honolulu, Hawaii, USA, Aug. 17-20, 1998). PACMEDTek '98. IEEE Computer Society, 344-352.

[3] Botsis, T. and Hartvigsen, G. Current Status and Future Perspectives in Telecare for Elderly People Suffering from Chronic Diseases. Journal of Telemedicine and Telecare 14, 4 (2008), 195-203.

[4] Jovanov, E., O'Donnell Lords, A., Raskovic, D., Cox, P. G., Adhami, R. and Andrasik, F. Stress Monitoring Using a Distributed Wireless Intelligent Sensor System. IEEE Engineering in Medicine and Biology Magazine 22, 3 (2003), 49-55.

[5] Jovanov, E. Wireless Technology and System Integration in Body Area Networks for m-Health Applications. In Proceedings of the 27th Annual International Conference of the Engineering in Medicine and Biology Society (Shanghai, China, Sept. 1-4, 2005). IEEE-EMBC '05. IEEE, 7158-7160.

[6] Kareem, S. and Bajwa, I. S. A Virtual Telehealth Framework: Applications and Technical Considerations. In Proceedings of the 7th International Conference on Emerging Technologies (Islamabad, Pakistan, Sept. 5-6, 2011). ICET '11. IEEE, 1-6.

[7] Dey, A. K. Understanding and Using Context. Personal Ubiquitous Comput. 5, 1 (2001), 4-7.

[8] Najar, S., Saidani, O., Kirsch-Pinheiro, M., Souveyet, C. and Nurcan, S. Semantic Representation of Context Models: A Framework for Analyzing and Understanding. In Proceedings of the 1st Workshop on Context, Information and Ontologies (Heraklion, Greece, 2009). ACM, 1-10.

[9] Suraci, V., Mignanti, S. and Aiuto, A. Context-aware Semantic Service Discovery. In Proceedings of the 16th IST Mobile and Wireless Communications Summit (Budapest, Hungary, Jul. 1-5, 2007). IEEE, 1-5. 